Technical note

\title{
Dose-escalated volumetric modulated arc therapy for total marrow irradiation: A feasibility dosimetric study with 4DCT planning and simultaneous integrated boost
}

\author{
Maud Jaccard $^{\mathrm{a}, *}$, Giorgio Lamanna ${ }^{\mathrm{a}}$, Melpomeni Kountouri ${ }^{\mathrm{a}}$, Michel Rouzaud ${ }^{\mathrm{a}}$, \\ Raymond Miralbell $^{\mathrm{a}, \mathrm{c}}$, Thomas Zilli ${ }^{\mathrm{a}, \mathrm{b}}$ \\ ${ }^{a}$ Radiation Oncology, University Hospital of Geneva, Geneva, Switzerland \\ ${ }^{\mathrm{b}}$ Faculty of Medicine, Geneva University, Geneva, Switzerland \\ ${ }^{\mathrm{c}}$ Radiation Oncology, Teknon Oncologic Institute, Quironsalud, Barcelona, Spain
}

\section{A R T I C L E I N F O}

\section{Keywords:}

Total marrow irradiation

Volumetric arc therapy

Simultaneous integrated boost

Planning organs at risk volume

Leukemia

\begin{abstract}
A B S T R A C T
Purpose: To evaluate the planning feasibility of dose-escalated total marrow irradiation (TMI) with simultaneous integrated boost (SIB) to the active bone marrow (ABM) using volumetric modulated arc therapy (VMAT), and to assess the impact of using planning organs at risk (OAR) volumes (PRV) accounting for breathing motion in the optimization.

Methods: Five patients underwent whole-body CT and thoraco-abdominal 4DCT. A planning target volume (PTV) including all bones and ABM was contoured on each whole-body CT. PRV of selected OAR (liver, heart, kidneys, lungs, spleen, stomach) were determined with 4DCT. Planning consisted of 9-10 full 6 MV photon VMAT arcs. Four plans were created for each patient with 12 Gy prescribed to the PTV, with or without an additional 4 Gy SIB to the ABM. Planning dose constraints were set on the OAR or on the PRV. Planning objective was a PTV $D_{\text {mean }}<110 \%$ of the prescribed dose, a PTV $V_{110 \%}<50 \%$, and OAR $D_{\text {mean }} \leq 50-60 \%$.

Results: PTV $\mathrm{D}_{\text {mean }}<110 \%$ was accomplished for most plans ( $\mathrm{n}=18 / 20$ ), while all achieved $\mathrm{V}_{110 \%}<50 \%$. SIB plans succeeded to optimally cover the boost volume (median $\mathrm{ABM} \mathrm{D}_{\text {mean }}=16.3 \mathrm{~Gy}$ ) and resulted in similar OAR sparing compared to plans without SIB (median OAR $\mathrm{D}_{\text {mean }}=40-54 \%$ of the ABM prescribed dose). No statistically significant differences between plans optimized with constraints on OAR or PRV were found. Conclusions: Adding a 4 Gy SIB to the ABM for TMI is feasible with VMAT technique, and results in OAR sparing similar to plans without SIB. Setting dose constraints on PRV does not impair PTV dosimetric parameters.
\end{abstract}

\section{Introduction}

Total body irradiation (TBI), associated to supra-lethal doses of chemotherapeutic agents, plays an essential role in the conditioning regimen for bone marrow transplantation (BMT) in patients with hematologic malignancies [1-5]. Slow-cycling bone marrow hematopoietic bone marrow stem cells may have limited radiosensitivity but a relatively high sensitivity to fractionation changes, favoring single-dose TBI or dose escalated protocols [6,7]. There is concern that the dose needed for their eradication in order to improve relapse-free survival figures might result in unacceptable toxicity with standard TBI [8-11]. Of note, randomized trials have shown decreased relapse rates in patients with acute myelogenous leukemia (AML) and chronic myelogenous leukemia (CML) with higher TBI dose $[8,9,12]$. Overall survival, however, has remained unchanged because of increased toxicity and treatment related mortality.

The implementation of modern radiotherapy (RT) techniques such as intensity-modulated RT (IMRT) or volumetric modulated arc therapy (VMAT) has introduced the possibility of more targeted forms of TBI, referred to as total marrow (TMI), total lymphoid (TLI) or combined total marrow and lymphoid irradiation (TMLI). Various studies have demonstrated the feasibility of using IMRT or helical tomotherapy (HT) for TMI, and their benefit for dose sparing of organs at risk (OAR) [13-21]. Moreover, dosimetric comparative studies showed that the use of VMAT could further improve the dose sparing of some OAR and significantly decrease the delivery time compared to IMRT or HT [22-26]. In addition to contributing in OAR sparing, the use of more conformal delivery techniques allows dose escalation in (or in selected

\footnotetext{
* Corresponding author at: Radiation Oncology Department, University Hospital of Geneva, 53 Av. de la Roseraie, 1205 Geneva, Switzerland.

E-mail address: maud.jaccard@hcuge.ch (M. Jaccard).
} 
parts of) the target volume as a way to improve survival figures.

The accurate delivery of conformal RT techniques is closely dependent on the correct patient daily repositioning at linac. Modern image-guided RT and efficient immobilization devices are therefore essential to reduce interfraction motion $[27,28]$. However, to treat tumors the thoraco-abdominal region, it is also crucial to take into account the intrafraction organ motion related to patient breathing.

The aim of this pilot study was to evaluate the treatment planning feasibility of a dose-escalated TMI program with simultaneous integrated boost (SIB) to the active bone marrow (ABM) using a VMAT technique. The hypothesis prompting this dosimetric investigation was that delivering a higher dose to the metabolically active part of the marrow could help to improve event-free survival in TMI patients. We intended to compare optimized treatment plans with and without SIB in terms of planning target volume (PTV) dosimetric parameters and OAR sparing. Furthermore, using 4DCT imaging to determine the planning at risk volumes (PRV) of the main OAR in the thoraco-abdominal region, we evaluated the dosimetric impact of sparing the PRV rather than the OAR in the plan optimization. This is the first dosimetric study investigating the feasibility of VMAT TMI including a SIB and the first attempt to set dosimetric constraints on PRV as a way to take into account the OAR breathing motion during treatment delivery.

\section{Materials and methods}

Five adult patients with hematological malignancies and candidates for conditioning with TBI before allogenic stem cell transplantation were enrolled in this study. All patients provided a written informed consent and the study was approved by the local ethical committee (CER: 13-138). For treatment planning purposes, all patients underwent a simulation procedure lying supine, arms alongside the body, and ankles placed in a Combifix device (Civco Medical Solutions, Orange City, USA). Thermoplastic contentions to immobilize the head and neck and the pelvic region were not used as this was a dosimetric study. Two CT sets of images were acquired for each patient: a whole-body freebreathing CT (WBFB CT) from top of the head to the mid-thigh with $5 \mathrm{~mm}$ axial slice thickness, and a 4DCT of the thoraco-abdominal region with $3 \mathrm{~mm}$ axial slice thickness.

Contouring was performed in the corresponding module of the treatment planning system (TPS) Eclipse version 10.0 (Varian Medical Systems, Palo Alto, USA). The clinical target volume (CTV) included the skeleton from the top of the head to mid-femurs, excluding the mandible, maxillary bones and hands. Isolated bones were contoured as a single connected outline, more specifically the ribs, the clavicles, the shoulders and the forearms [22]. The planning target volume (PTV) was defined by adding a 3-mm isotropic expansion to the CTV following guidelines of similar TMI planning studies [14-16,20]. For the plans including a dose escalation with a SIB, the active bone marrow (ABM) volume was defined as the lumbar and thoracic spine as well as the pelvis, except for the sacrum, since the former contain approximately $60 \%$ of all active bone marrow [29]. A $1-\mathrm{mm}$ external wall was subtracted from the above mentioned bone structure to obtain the final ABM volume (Fig. 1). No extra PTV margin was added.

The OAR included the brain, eyes, optic nerves, lenses, parotids, oral cavity, larynx, thyroid, lungs, heart, esophagus, liver, spleen, stomach, kidneys, bladder, rectum, uterus and vagina, prostate and external genitals. OAR were defined according to the RTOG guidelines when available. Selected OAR in the thoraco-abdominal region (heart, liver, lungs, kidneys, spleen, and stomach) were contoured on the 10 respiratory phases of the 4DCT. For each of these OAR, a PRV was created on the WBFB CT by accumulation of the OAR contours outlined on the 4DCT phases.

Treatment plans were created on the WBFB CT in Eclipse, optimized with the Progressive Resolution Optimizer (PRO v.10.0) and calculated with Analytical Anisotropic Algorithm AAA algorithm (v.10.0) with a calculation grid size of $2.5 \mathrm{~mm}$. The prescribed dose to the PTV was

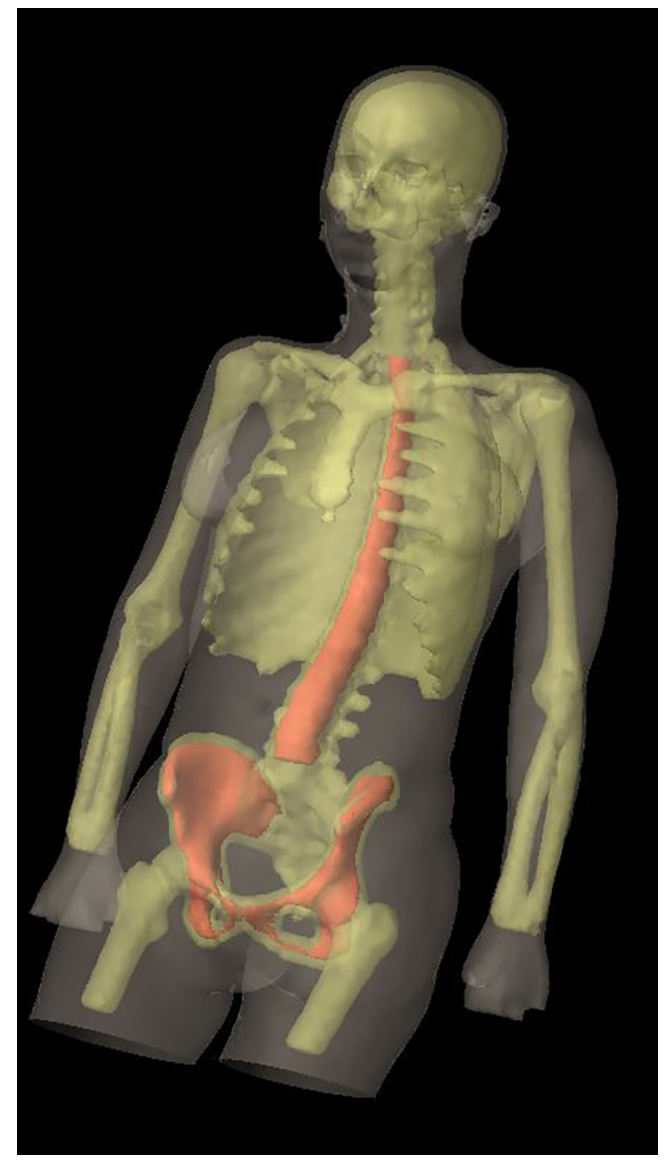

Fig. 1. Clinical target volume outline for total marrow irradiation to $12 \mathrm{~Gy}$ (yellow outline) with a simultaneous integrated boost to $16 \mathrm{~Gy}$ to the active bone marrow (red outline), defined as the lumbar and thoracic spine as well as the pelvis, except for the sacrum. (For interpretation of the references to color in this figure legend, the reader is referred to the web version of this article.)

$12 \mathrm{~Gy}$ delivered in 6 fractions of $2 \mathrm{~Gy}$. For the SIB technique, $16 \mathrm{~Gy}$ in 6 fractions of $2.67 \mathrm{~Gy}$ were planned to be delivered to the ABM simultaneously to the standard dose prescription to the PTV.

Two different plans were created for each prescription with different optimization strategies on OAR. For the VMAT and VMAT-SIB plans, we set the optimization dose constraints on the OAR, while for $V M A T-P R V$ and VMAT-PRV-SIB, dose constraints were applied to the PRV. The plans without SIB were created before those with SIB, and the same dose values constraints and weights were used for OAR and PRV in the optimization objectives of the plans with or without SIB. This resulted in four treatment plans per patient. All plans were created and optimized by the same physicist (MR).

We planned to treat with 9 to 10 adjacent full VMAT coplanar arcs of 6 MV photon beams arranged along the patient's longitudinal axis, with a $10 \mathrm{~cm}$ distance between each isocentre, in a similar way as described by Fogliata et al. [22]. All isocenters had the same lateral and anteroposterior (AP) coordinates, hence for the treatment only longitudinal shifts would be needed. There was an overlap region between arcs of approximately $4 \mathrm{~cm}$, depending on patient's size. The collimator angle was set to $90^{\circ}$ for all arcs, with jaws set to $40 \mathrm{~cm}$ (y-axis) and $14 \mathrm{~cm}$ (x-axis). Plans were calculated to be delivered with a Clinac $2300 \mathrm{iX}$ (Varian Medical Systems) mounted with a Millenium ${ }^{\mathrm{TM}}$ multileaf collimator, with leaves of $5 \mathrm{~mm}$ width in the central region $(20 \mathrm{~cm} \times 20 \mathrm{~cm})$ and of $10 \mathrm{~mm}$ in the outer part. We used the maximum available dose-rate of $600 \mathrm{MU} / \mathrm{min}$.

All plans were normalized such that $100 \%$ of the prescribed dose was delivered to at least $85 \%$ of the PTV $\left(\mathrm{D}_{85 \%}=100 \%\right.$ or $\mathrm{V}_{100 \%}=$ 
85\%) in agreement with published TMI planning studies [20,22,23,30]. Planning aimed to achieve a PTV $\mathrm{D}_{\text {mean }}<110 \%$ of the prescribed dose (13.2 Gy), a $\mathrm{V}_{110 \%}<50 \%$ and a $\mathrm{D}_{1 \%}$ as low as reasonably achievable. In analogy with other TMI studies $[13,16,18,22,23]$ for the OAR, the present dosimetric study required $D_{\text {mean }} \leq 50-60 \%$ of the prescription dose (i.e., 6 to $7.2 \mathrm{~Gy}$ ) in order to half the dose received by the same OAR with a TBI and the same prescribed dose.

PTV and ABM volumes as well as the cranio-caudal PTV length were extracted from the WBFB CT for all patients. In the present study, we focused our analysis on the main OAR of the thoraco-abdominal region (heart, liver, lungs, kidneys, spleen, and stomach). OAR and PRV volumes were compared. OAR $\mathrm{D}_{\text {mean }}$ in each of the four plans were extracted from the TPS and differences between groups analyzed.

Statistical analyses were carried out using the software SPSS (v.24, IBM, Chicago, USA). Significance levels for the difference between dosimetric parameters (plans with or without SIB and plans with or without PRV) were determined using the non-parametric related-samples Wilcoxon signed-rank test, with $p$-values $\leq 0.05$ considered as statistically significant.

All patients in this dosimetric study were treated with standard TBI using a pair of AP-posteroanterior $6 \mathrm{MV}$ photon opposed fields with adequate lung shielding as routinely performed in our department $[31,32]$.

\section{Results}

Table 1 shows median PTV and ABM volumes, as well as the PTV length of the five patients included in this study. For the patient with the shortest PTV length (i.e. $88.4 \mathrm{~cm}$ ), we used only 9 isocenters for the planning, while the treatment plans of the other four patients required 10 isocenters. OAR and PTV volumes as contoured on the WBFB are reported in Table 2. Using a 4DCT to create PRV, the average median volume of OAR contoured on WBFB CT increased by $27 \%$. The smallest relative median increase occurred for the liver $(10 \%)$ and the largest for the heart (39\%) (Table 2).

All plans were normalized to ensure that the prescribed dose of 12 Gy covered at least $85 \%$ of the PTV. The goal of delivering to the PTV a $\mathrm{D}_{\text {mean }}<13.2 \mathrm{~Gy}$ was respected for all plans but two (for which PTV $D_{\text {mean }}$ was $13.3 \mathrm{~Gy}$ ). Furthermore, all plans achieved the goal of a $\mathrm{V}_{110 \%}<50 \%$ (Table 3 ). The SIB plans succeeded to cover the ABM with a $\mathrm{D}_{\text {mean }}$ close to the prescribed dose of $16 \mathrm{~Gy}$, as well as a $\mathrm{V}_{100 \%}$ ranging between 60 and $80 \%$ of the ABM volume. Regarding the OAR, the constraint $\mathrm{D}_{\text {mean }}<50-60 \%$ was respected in most cases for the heart, kidneys and stomach. There was less compliance for the liver and the spleen $\left(D_{\text {mean }} \approx 65 \%\right.$ ), and for the lungs ( $D_{\text {mean }} \approx 70-75 \%$ ) (Table 3 ).

Fig. 2 illustrates the color-wash dose distribution for the standard dose TMI plan (12 Gy) (a) compared to a dose-escalated TMI (16 Gy to the ABM) (b). Median PTV $D_{\text {mean }}$ and $V_{110 \%}$ were similar in plans with or without SIB (Table 3 ), while $\mathrm{D}_{1 \%}$ was higher in plans with SIB in accordance with the prescription. Surprisingly, VMAT-SIB plans resulted in better OAR sparing than plans without SIB, with a statistically

Table 1

Geometric characteristics of target volumes for TMI treatment planning.

\begin{tabular}{ll}
\hline & $\begin{array}{l}\text { Median } \\
\text { [min-max] }\end{array}$ \\
\hline PTV volume $\left(\mathrm{cm}^{3}\right)$ & 6828.1 \\
& {$[5982.4-8921.5]$} \\
PTV length $(\mathbf{c m})$ & 93.0 \\
& {$[88.4-99.1]$} \\
ABM volume $\left(\mathrm{cm}^{3}\right)$ & 660.1 \\
& {$[576.3-965.8]$}
\end{tabular}

Abbreviations: TMI: total marrow irradiation; PTV: planning target volume; ABM: active bone marrow.
Table 2

Median [min-max] volume of OAR from free-breathing CT scan and PRV from 4DCT scan, and median volume increase from OAR to PRV.

\begin{tabular}{|c|c|c|c|c|}
\hline & OAR volume $\left(\mathrm{cm}^{3}\right)$ & PRV volume $\left(\mathrm{cm}^{3}\right)$ & $\begin{array}{l}\text { Median } \\
\text { volume } \\
\text { increase } \\
\text { (\%) }\end{array}$ & $\begin{array}{l}\text { Median } \\
\text { volume } \\
\text { increase } \\
\left(\mathrm{cm}^{3}\right)\end{array}$ \\
\hline Heart & $\begin{array}{l}481.4 \\
{[399.0-708.6]}\end{array}$ & $\begin{array}{l}604 \\
{[494.7-984.2]}\end{array}$ & 38.9 & 193.0 \\
\hline Liver & $\begin{array}{l}1582.1 \\
{[1119.2-2421.1]}\end{array}$ & $\begin{array}{l}1745.34 \\
{[1198.4-2542.1]}\end{array}$ & 10.3 & 163.3 \\
\hline Lungs & $\begin{array}{l}2398.0 \\
{[1651.7-2986.3]}\end{array}$ & $\begin{array}{l}2441 \\
{[2045.1-3946.5]}\end{array}$ & 12.0 & 325.0 \\
\hline Kidneys & $\begin{array}{l}337.1 \\
{[233.9-353.6]}\end{array}$ & $\begin{array}{l}419.28 \\
{[301.5-453.1]}\end{array}$ & 29.0 & 88.9 \\
\hline Spleen & $\begin{array}{l}137.5 \\
{[57.5-991.6]}\end{array}$ & $\begin{array}{l}227.7 \\
{[79.8-995.6]}\end{array}$ & 34.3 & 30.7 \\
\hline Stomach & $\begin{array}{l}443.3 \\
{[140.6-668.7]}\end{array}$ & $\begin{array}{l}612.3 \\
{[271.4-790.7]}\end{array}$ & 38.1 & 161.0 \\
\hline
\end{tabular}

Abbreviations: OAR: organs at risk; PRV: planning organs at risk volume.

significant reduction $(-6 \%)$ in median doses to the OAR (Table 3). The number of monitor units (MU) was 33\% larger in plans with SIB, as expected since the delivery of a higher dose requires in general more MU.

Finally, our data show that performing the optimization with constraints set on the PRV did not negatively impact the PTV or ABM dosimetric results. Indeed there were no statistically significant differences when comparing the plans VMAT $v$ s. VMAT-PRV and VMAT-SIB vs. VMAT-PRV-SIB, both for the target volumes (Table 2 and Fig. 3) and for the OAR (Table 2).

\section{Discussion}

TBI is a key component in the treatment management of patients with hematologic malignancies and studies have shown that higher dose may be associated with a reduced post-transplant relapse probability [12]. However, the dose that can be delivered by TBI is limited by toxicity. More targeted irradiation techniques increasing the dose delivered to the target and sculpting the dose around the OAR can represent a treatment strategy able to improve the therapeutic ratio in patients who are candidates for BMT.

Emerging TMI techniques with HT [16-18,20,33] or fixed gantry linac IMRT $[13,15]$ have reported substantial reduction of normal tissue dose relative to conventional TBI, with median doses to OARs about $15-65 \%$ of the prescribed target volume dose. Early clinical results have suggested a reduced risk of acute toxicity in patients with targeted TBI (TMI, TLI, TMLI) compared to those receiving standard TBI and other standard conditioning regimens [20,34-36]. In addition, HT has also been used to further increase the dose to the total bone marrow after a standard TBI, a treatment which was associated with a low rate of severe toxicity [37].

In the present study, we undertook a dosimetric optimization effort for five patients planned with VMAT for TMI with or without an SIB to the ABM. Results on this small sample showed that adding an SIB to the ABM did not negatively impact the PTV dose in terms of $D_{\text {mean }}$ and $\mathrm{V}_{110 \%}$ while achieving a fair sparing of OAR in the thoracic region. Patients in this study underwent a 4DCT in order to define, for selected OAR, their respective PRV in the thoraco-abdominal region, thus helping to account for possible organs motion during treatment. Treatment planning with or without SIB was repeated, setting dose constrains on the PRV instead of the OAR during the optimization process. There was no dose distribution impairment to the PTV when setting the constraints on PRV.

The role of a VMAT for TMI was investigated in several publications $[22,23]$. Stated OAR doses were slightly reduced compared to HT and ranged between 5 and $50 \%$ of the prescribed dose. Moreover the beam- 
Table 3

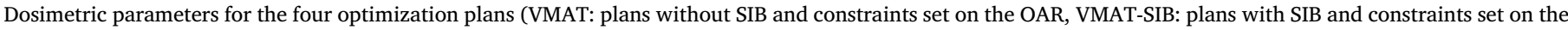

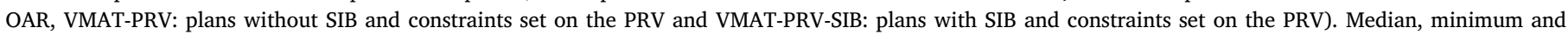
maximum values are presented. The * indicate medians which are significantly different than the corresponding median for the plan without SIB.

\begin{tabular}{|c|c|c|c|c|c|}
\hline & & VMAT & VMAT-SIB & VMAT-PRV & VMAT-PRV-SIB \\
\hline \multirow[t]{6}{*}{ PTV } & $D_{\text {mean }}(G y)$ & 12.9 & 13.2 & 12.9 & 13.3 \\
\hline & & [12.8-13.3] & [13.0-13.2] & [12.8-13.0] & [13.2-13.3] \\
\hline & $\mathrm{V}_{110 \%}(\%)$ & 37.6 & 33.9 & 41.4 & 35.5 \\
\hline & & [29.7-48.8] & [30.0-34.6] & [32.4-47.3] & [33.0-38.0] \\
\hline & $D_{1 \%}(G y)$ & 14.3 & $17.2^{*}$ & 14.5 & $17.3^{*}$ \\
\hline & & [14.0-15.2] & [15.7-17.3] & [14.2-14.7] & [17.0-17.5] \\
\hline \multirow[t]{6}{*}{ ABM } & $D_{\text {mean }}(G y)$ & NA & 16.3 & NA & 16.4 \\
\hline & & & [16.2-16.5] & & [16.3-16.5] \\
\hline & $\mathrm{V}_{100 \%}(\%)$ & NA & 62.0 & NA & 69.0 \\
\hline & & & [60.1-80.0] & & [61.7-80.5] \\
\hline & $\mathrm{V}_{110 \%}(\%)$ & NA & 0.1 & NA & 0.9 \\
\hline & & & [0.0-1.3] & & {$[0.1-5.1]$} \\
\hline OAR & Heart & 7.1 & $6.6 *$ & 6.8 & $6.4^{*}$ \\
\hline \multirow[t]{13}{*}{$D_{\text {mean }}(G y)$} & & [6.9-7.9] & [6.5-6.7] & & [6.4-6.6] \\
\hline & & & & [6.8-7.2] & \\
\hline & Liver & 8.0 & $7.5^{*}$ & 8.0 & $7.4^{*}$ \\
\hline & & [7.7-8.1] & {$[7.2-7.6]$} & & [7.0-7.8] \\
\hline & & & & [7.7-8.4] & \\
\hline & Lungs & 9.1 & $8.6^{*}$ & 9.1 & $8.6^{*}$ \\
\hline & & [8.8-9.4] & [8.3-8.7] & [8.8-9.2] & [8.3-8.7] \\
\hline & Kidneys & 7.3 & $7.0 *$ & 7.2 & $6.8^{*}$ \\
\hline & & [6.9-7.5] & {$[6.2-7.0]$} & [6.9-7.5] & [6.4-6.9] \\
\hline & Spleen & 8.2 & $7.7^{*}$ & 8.0 & $7.5^{*}$ \\
\hline & & [8.0-8.5] & [7.6-7.7] & [7.9-8.4] & [7.2-8.0] \\
\hline & Stomach & 6.8 & $6.4 *$ & 7.2 & $6.7 *$ \\
\hline & & [6.7-7.4] & {$[6.3-6.6]$} & [6.9-9.8] & [5.9-6.8] \\
\hline \multirow[t]{2}{*}{ Total MU number } & & 2755 & $3513^{*}$ & 2694 & $3518^{*}$ \\
\hline & & [2484-2775] & [3147-3662] & [2421-2778] & [3191-3631] \\
\hline
\end{tabular}

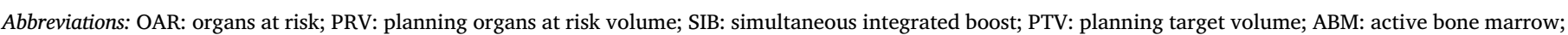
$\mathrm{D}_{\text {mean }}$ : mean dose received by the volume; $\mathrm{V}_{\mathrm{X} \%}$ volume receiving $\mathrm{X} \%$ of the prescribed dose; $\mathrm{D}_{1 \%}$ : dose received by $1 \%$ of the volume; MU: monitor units.

on time with VMAT was reported to be less than 20 min (e.g. 11, 13, and $18 \mathrm{~min}$ reported by Han et al. [24], Fogliata et al. [22] and Aydogan et al. [23], respectively), while a wider range of beam-on times was obtained with HT, from 16 [19] to $50 \mathrm{~min}[18,20]$. The dose to the OAR reported in the literature is, on average, inferior to the dose to the respective OAR in our dosimetric study (Table 1). This might be explained by differences in the definition of PTVs and/or in planning techniques across studies [22,23], as well as by inherent variations in patient's anatomy. This variability, in addition to the relatively small sample size of TMI planning studies, could significantly flaw the comparison with results obtained by other groups.
Although being based on a small patient sample, our results seem to suggest that dose-escalated TMI with a SIB up to 16 Gy to the ABM is feasible and reliable without increasing the dose received by the OAR, and even with a slight dose reduction. The reason for this dose reduction is not clear but might be due to particular implementation in the optimization algorithm of the treatment planning system. The therapeutic benefit from increasing the dose per fraction ( $>2 \mathrm{~Gy}$ ) is suggested by clinical and radio-biological data highlighting that radiation dose and fractionation have a major impact in determining the success of long term marrow engraftment. The fact that the primitive self-renewing stem cells population of the host marrow displays a significant
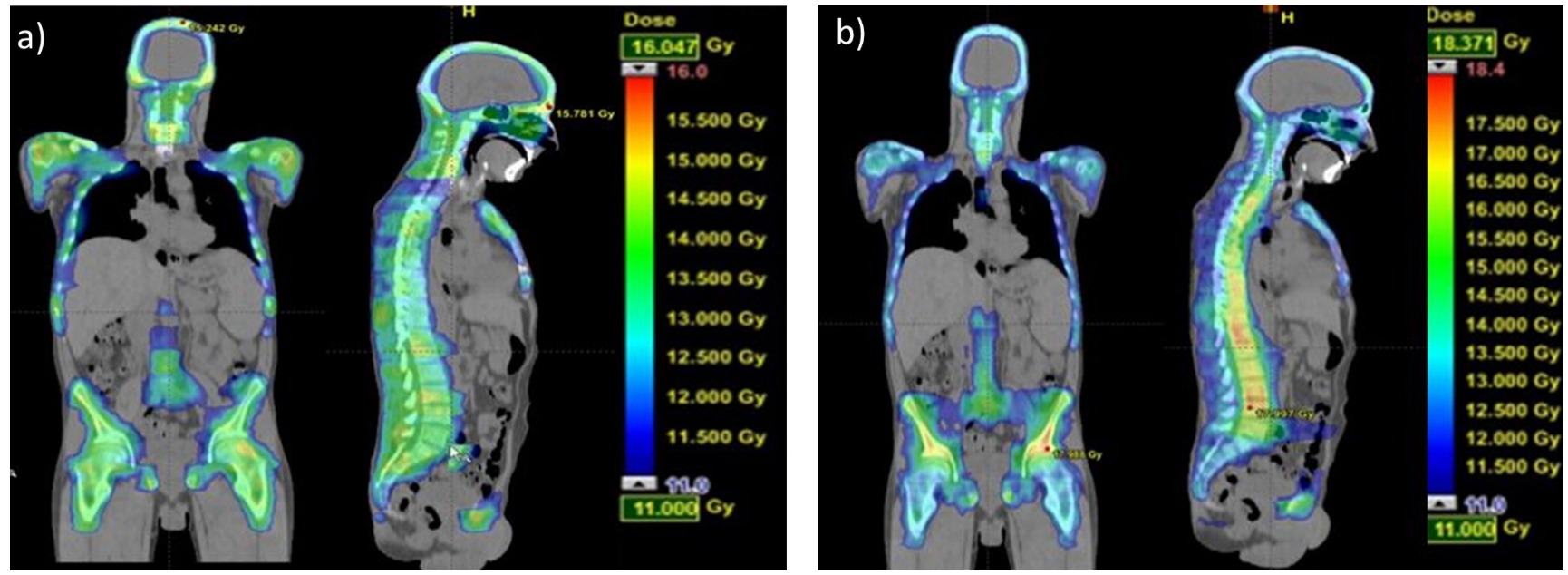

Fig. 2. An example of dose distribution for one of the patients with a) standard dose TMI (12 Gy) compared to b) a dose-escalated TMI (16 Gy to the ABM) on coronal and sagittal views. Abbreviation: TMI: total marrow irradiation, ABM: active bone marrow. 

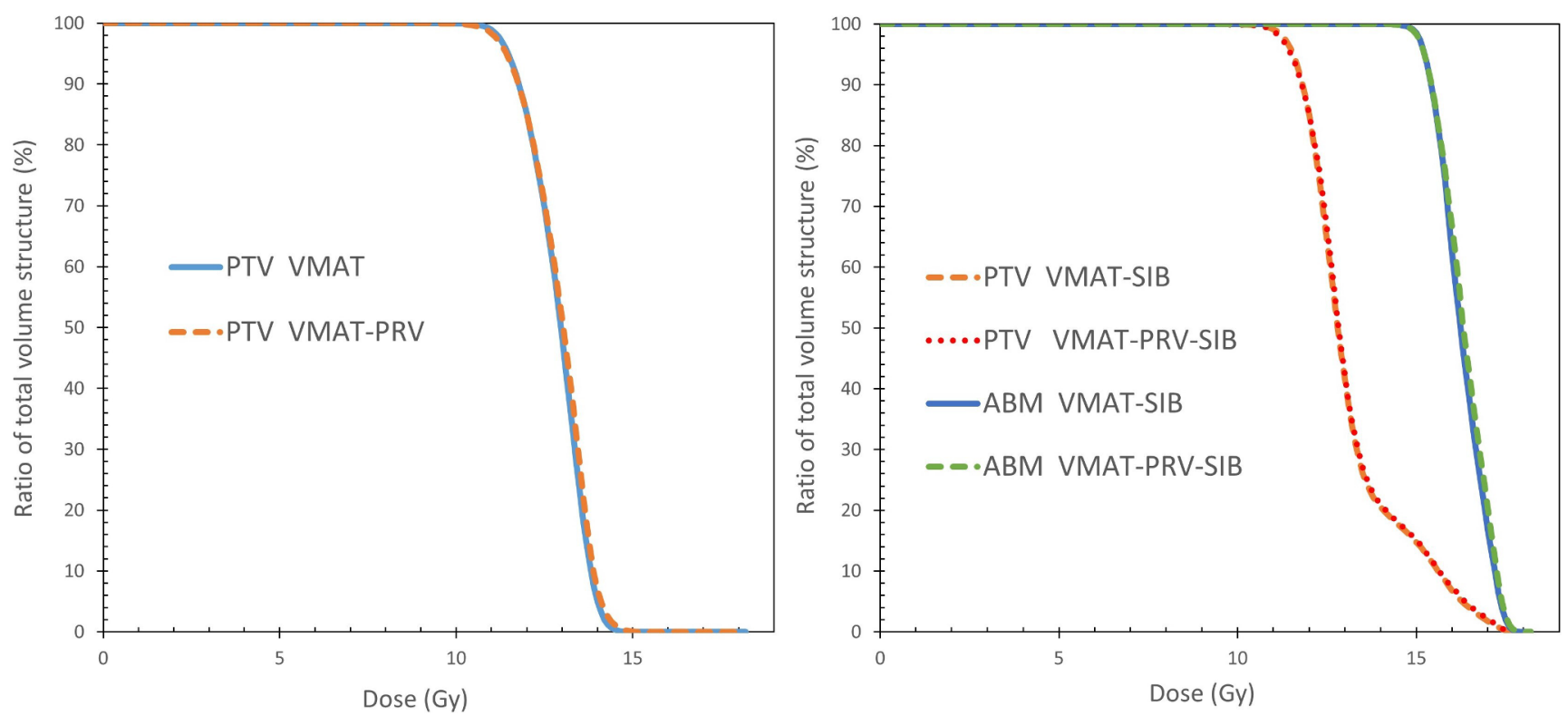

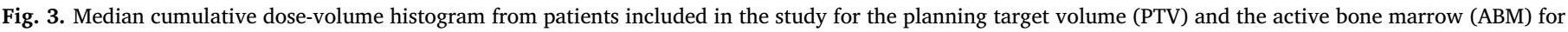

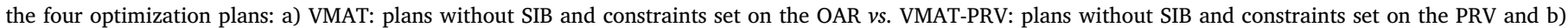
VMAT-SIB: plans with SIB and constraints set on the OAR vs. VMAT-PRV-SIB: plans with SIB and constraints set on the PRV.

sub-lethal damage repair, favors the option of a hypofractionated schedule $[6,7,38]$ to improve tumor control. Indeed, it was shown that patients with CML who received fractionated TBI had a higher risk of relapse $[39,40]$ than those treated with a single fraction, suggesting a role for hypofractionated regimens in this group of patients.

In this study, the volume receiving the escalated dose was defined according to the study by Hayman et al. [29], which shows that it contained approximatively $60 \%$ of all active bone marrow. According to this, the hypothesis at the origin of our dosimetric research was that delivering a boost to the most metabolically active bone marrow regions might translate into a lower relapse rate in patients after undergoing a TMI. On the other hand, 18F-fluoro-L-deoxythymidine (FLT)PET imaging could be recommended to identify the specific metabolic ABM distribution in an individualized patient. In addition, Magome et al. $[41,42]$ employed FLT-PET and dual-energy CT to identify leukemia distribution within the bone marrow. Using treatment planning with HT, they showed that the dose could be escalated in these regions by 2 -fold, while maintaining OAR doses at the same level as in standard TMI.

We were unable to observe a negative impact on dose-distribution quality indicators of the target volumes (PTV and ABM) when sparing PRV instead of OAR. Furthermore, a PRV-based optimization taking into account intrafraction motion due to breathing might have more chances to succeed in optimally matching dose calculations $v s$. dose measurements for the OAR. Nevertheless, PRV delineation requires 4DCT images and contouring on each phase of the respiratory cycle, a time-consuming task. This burden will probably be alleviated in the future with oncoming efficient auto-segmentation tools [43,44]. An alternative solution for the motion management of TMI treatment is respiratory gating to deliver the treatment to a specific breathing phase, with the advantage of an even better targeting of the PTV, but with the drawback of prolonging the treatment time of an already long treatment. Such gating could be potentially implemented with a surface monitoring system, expected to facilitate also the patient setup, in particular for the repositioning of the extremities, and the intrafractional motion control during the relatively long TMI treatment times.

This was a dosimetric feasibility study with a limited number of five patients all simulated for the purpose of the study and that were treated with standard TBI. This reduced number of patients prevents drawing robust and reliable dosimetric conclusions apart from the feasibility of the technique similar to other TMI planning studies $[13,15,17,20,22-24]$. Similarly, significance of the statistical results should be interpreted with care, keeping in mind the small sample size. The irradiation of the lower limbs, usually treated with a series of VMAT arcs (or simple open fields) and the patient in feet-first orientation, was not included in our analysis similarly to other TMI planning studies $[13,14,16]$. In practice, this would add the challenge of managing a treatment involving two plans with different treatment orientations $[45,46]$.

\section{Conclusion}

In summary, our preliminary study seems to suggest that adding a dose of 4 Gy (ABM-SIB) to a 12 Gy TMI using VMAT techniques should be feasible without any clinically relevant dose increase to the OAR. Further investigations to better quantify the impact of ABM-SIB is warranted. Furthermore, PRV defined from 4DCT could be reliably used without relevant PTV dosimetry drawbacks.

\section{Funding}

The implementation of this project has been funded by a grant of the Fondation Dr Henri Dubois-Ferrière Dinu Lipatti and by a STARTUP and a PRD (projet de recherche et développement) grants of the University Hospital of Geneva.

\section{Declaration of Competing Interest}

The authors declare that they have no known competing financial interests or personal relationships that could have appeared to influence the work reported in this paper.

\section{References}

[1] Thomas ED, Storb R, Clift RA, Fefer A, Johnson FL, Neiman PE, Lerner KG, Glucksberg H, Buckner CD. Bone-marrow transplantation. N Engl J Med 1975;292(16):832-43.

[2] Ringden O, Ruutu T, Remberger M, Nikoskelainen J, Volin L, Vindelov L, et al. A randomized trial comparing busulfan with total body irradiation as conditioning in allogeneic marrow transplant recipients with leukemia: a report from the Nordic Bone Marrow Transplantation Group. Blood. 1994;83:2723. 
[3] Dusenbery KE, Daniels KA, McClure JS, McGlaver PB, Ramsay NKC, Blazar BR, Neglia JP, Kersey JH, Woods WG. Randomized comparison of cyclophosphamidetotal body irradiation versus busulfan-cyclophosphamide conditioning in autologous bone marrow transplantation for acute myeloid leukemia. Int J Radiation Oncol*Biol*Phys 1995;31(1):119-28.

[4] Blaise D, Maraninchi D, Michallet M, Reiffers J, Jouet JP, Milpied N, et al. Longterm follow-up of a randomized trial comparing the combination of cyclophosphamide with total body irradiation or busulfan as conditioning regimen for patients receiving HLA-identical marrow grafts for acute myeloblastic leukemia in first complete remission. Blood. 2001;97:3669.

[5] Bunin N, Aplenc R, Kamani N, Shaw K, Cnaan A, Simms S. Randomized trial of busulfan vs total body irradiation containing conditioning regimens for children with acute lymphoblastic leukemia: a pediatric blood and marrow transplant consortium study. Bone Marrow Transplant 2003;32(6):543-8.

[6] Cosset J-M, Socie G, Dubray B, Girinsky T, Fourquet A, Gluckman E. Single dose versus fractionated total body irradiation before bone marrow transplantation: Radiobiological and clinical considerations. Int J Radiation Oncol*Biol*Phys 1994;30(2):477-92.

[7] Down JD, Tarbell NJ, Thames HD, Mauch PM. Syngeneic and allogeneic bone marrow engraftment after total body irradiation: dependence on dose, dose rate, and fractionation. Blood. 1991;77:661-9.

[8] Clift RA, Buckner CD, Appelbaum FR, Bearman SI, Petersen FB, Fisher LD, et al. Allogeneic marrow transplantation in patients with acute myeloid leukemia in first remission: a randomized trial of two irradiation regimens. Blood 1990;76:1867-71.

[9] Clift RA, Buckner CD, Appelbaum FR, Bryant E, Bearman SI, Petersen FB, et al Allogeneic marrow transplantation in patients with chronic myeloid leukemia in the chronic phase: a randomized trial of two irradiation regimens. Blood. 1991;77:1660-5.

[10] Clift RA, Buckner CD, Appelbaum FR, Sullivan KM, Storb R, Thomas ED. Long-Term Follow-Up of a Randomized Trial of Two Irradiation Regimens for Patients Receiving Allogeneic Marrow Transplants During First Remission of Acute Myeloid Leukemia. Blood. 1998;92:1455.

[11] Bieri S, Helg C, Chapuis B, Miralbell R. Total body irradiation before allogeneic bone marrow transplantation: is more dose better? Int J Radiation Oncol*Biol*Phys 2001;49(4):1071-7.

[12] Scarpati D, Frassoni F, Vitale V, Corvo R, Franzone P, Barra S, Guenzi M, Orsatti M. Total body irradiation in acute myeloid leukemia and chronic myelogenous leukemia: influence of dose and dose-rate on leukemia relapse. Int J Radiation Oncol*Biol*Phys 1989;17(3):547-52.

[13] Aydogan B, Mundt AJ, Roeske JC. Linac-Based Intensity Modulated Total Marrow Irradiation (IM-TMI). Technol Cancer Res Treat 2006;5(5):513-9.

[14] Wilkie JR, Tiryaki H, Smith BD, Roeske JC, Radosevich JA, Aydogan B. Feasibility study for linac-based intensity modulated total marrow irradiation: Linac-based intensity modulated total marrow irradiation. Med Phys 2008;35(12):5609-18.

[15] Yeginer M, Roeske JC, Radosevich JA, Aydogan B. Linear Accelerator-Based Intensity-Modulated Total Marrow Irradiation Technique for Treatment of Hematologic Malignancies: A Dosimetric Feasibility Study. Int J Radiation Oncol*Biol*Phys 2011;79(4):1256-65.

[16] Hui SK, Kapatoes J, Fowler J, Henderson D, Olivera G, Manon RR, et al. Feasibility study of helical tomotherapy for total body or total marrow irradiation. Med Phys 2005;32:3214-24.

[17] Wong JYC, Liu An, Schultheiss T, Popplewell L, Stein A, Rosenthal J, Essensten M, Forman S, Somlo G. Targeted Total Marrow Irradiation Using Three-Dimensional Image-Guided Tomographic Intensity-Modulated Radiation Therapy: An Alternative to Standard Total Body Irradiation. Biol Blood Marrow Transplantation 2006;12(3):306-15.

[18] Schultheiss TE, Wong J, Liu An, Olivera G, Somlo G. Image-guided total marrow and total lymphatic irradiation using helical tomotherapy. Int J Radiation Oncol*Biol*Phys 2007;67(4):1259-67.

[19] Hui SK, Verneris MR, Higgins P, Gerbi B, Weigel B, Baker SK, Fraser C, Tomblyn M, Dusenbery K. Helical tomotherapy targeting total bone marrow - First clinical experience at the University of Minnesota. Acta Oncol 2007;46(2):250-5.

[20] Wong JYC, Rosenthal J, Liu An, Schultheiss T, Forman S, Somlo G. Image-Guided Total-Marrow Irradiation Using Helical Tomotherapy in Patients With Multiple Myeloma and Acute Leukemia Undergoing Hematopoietic Cell Transplantation. Int J Radiation Oncol*Biol*Phys 2009;73(1):273-9.

[21] Zeverino M, Agostinelli S, Taccini G, Cavagnetto F, Garelli S, Gusinu M, Vagge S, Barra S, Corvò R. Advances in the implementation of helical tomotherapy-based total marrow irradiation with a novel field junction technique. Med Dosim 2012;37(3):314-20.

[22] Fogliata A, Cozzi L, Clivio A, Ibatici A, Mancosu P, Navarria P, Nicolini G, Santoro A, Vanetti E, Scorsetti M. Preclinical Assessment of Volumetric Modulated Arc Therapy for Total Marrow Irradiation. Int J Radiation Oncol*Biol*Phys 2011;80(2):628-36.

[23] Aydogan B, Yeginer M, Kavak GO, Fan J, Radosevich JA, Gwe-Ya K. Total Marrow Irradiation With RapidArc Volumetric Arc Therapy. Int J Radiation Oncol*Biol*Phys 2011;81(2):592-9.

[24] Han C, Schultheisss TE, Wong JYC. Dosimetric study of volumetric modulated arc therapy fields for total marrow irradiation. Radiother Oncol 2012;102(2):315-20.

[25] Mancosu P, Cozzi L, Muren LP. Total marrow irradiation for hematopoietic ma lignancies using volumetric modulated arc therapy: A review of treatment planning studies. Phys Imag Radiation Oncol 2019;11:47-53.

[26] Litoborska J, Piotrowski T, Malicki J. Evaluation of three VMAT-TMI planning methods to find an appropriate balance between plan complexity and the resulting dose distribution. Phys Med 2020;75:26-32.

[27] Mancosu P, Navarria P, Muren LP, Castagna L, Reggiori G, Clerici E, Sarina B, Bramanti S, De Philippis C, Tomatis S, Santoro A, Scorsetti M. Development of an Immobilization Device for Total Marrow Irradiation. Practical Radiation Oncol 2020. https://doi.org/10.1016/j.prro.2020.02.012.

[28] Zuro D, Vagge S, Broggi S, Agostinelli S, Takahashi Y, Brooks J, Leszcynska P, Liu An, Zucchetti C, Saldi S, Han C, Cattaneo M, Giebel S, Mahe MA, Sanchez JF, Alaei P, Anna C, Dusenbery K, Pierini A, Storme G, Aristei C, Wong JYC, Hui S. Multiinstitutional evaluation of MVCT guided patient registration and dosimetric precision in total marrow irradiation: A global health initiative by the international consortium of total marrow irradiation. Radiother Oncol 2019;141:275-82.

[29] Hayman JA, Callahan JW, Herschtal A, Everitt S, Binns DS, Hicks RJ, Mac Manus M. Distribution of Proliferating Bone Marrow in Adult Cancer Patients Determined Using FLT-PET Imaging. Int J Radiation Oncol*Biol*Phys 2011;79(3):847-52.

[30] Mancosu P, Navarria P, Castagna L, Roggio A, Pellegrini C, Reggiori G, Fogliata A, Lobefalo F, Castiglioni S, Alongi F, Cozzi L, Santoro A, Scorsetti M. Anatomy driven optimization strategy for total marrow irradiation with a volumetric modulated arc therapy technique. J Appl Clinical Med Phys 2012;13(1):138-47.

[31] Miralbell R, Rouzaud M, Grob E, Nouet P, Bieri S, Majno SB, Botteron P, Montero M, Precoma JC. Can a total body irradiation technique be fast and reproducible? Int J Radiation Oncol*Biol*Phys 1994;29(5):1167-73.

[32] Dipasquale G, Miralbell R, Lamanna G, Nouet P, Montero M, Rouzaud M, Zilli T. Image-guided total-body irradiation with a movable electronic portal imaging device for bone marrow transplant conditioning. Zeitschrift für Medizinische Physik 2020;30(2):148-54.

[33] Haraldsson A, Engellau J, Lenhoff S, Engelholm S, Bäck S, Engström PE. Implementing safe and robust Total Marrow Irradiation using Helical Tomotherapy - A practical guide. Phys Med 2019;60:162-7.

[34] Rosenthal J, Wong J, Stein A, Qian D, Hitt D, Naeem H, et al. Phase 1/2 trial of total marrow and lymph node irradiation to augment reduced-intensity transplantation for advanced hematologic malignancies. Blood. 2011;117:309-15.

[35] Somlo G, Spielberger R, Frankel P, Karanes C, Krishnan A, Parker P, Popplewell L, Sahebi F, Kogut N, Snyder D, Liu A, Schultheiss T, Forman S, Wong JYC. Total Marrow Irradiation: A New Ablative Regimen as Part of Tandem Autologous Stem Cell Transplantation for Patients with Multiple Myeloma. Clin Cancer Res 2011;17(1):174-82.

[36] Assanelli AA, Lorentino F, Marcatti M, Chiara A, Perini T, Lupo Stanghellini MT, et al. Escalated Dose-Rates of Total Marrow Irradiation (TMI) Combined with Treosulfan and Fludarabine-Based Conditioning Chemotherapy Regimen for Chemosensitive Advanced Multiple Myeloma (MM) Patients Undergoing a Matched Allogeneic Stem-Cell Transplantation: First Results of a Phase I/II Prospective Monocentric Study (TrRaMM TMI). Blood. 2016;128:2221.

[37] Corvò R, Zeverino M, Vagge S, Agostinelli S, Barra S, Taccini G, Lint MTV, Frassoni F, Bacigalupo A. Helical tomotherapy targeting total bone marrow after total body irradiation for patients with relapsed acute leukemia undergoing an allogeneic stem cell transplant. Radiother Oncol 2011;98(3):382-6.

[38] Corvò R, Lamparelli T, Bruno B, Barra S, Van Lint MT, Vitale V, Bacigalupo A. Lowdose fractionated total body irradiation (TBI) adversely affects prognosis of patients with leukemia receiving an HLA-matched allogeneic bone marrow transplant from an unrelated donor (UD-BMT). Bone Marrow Transplant 2002;30(11):717-23.

[39] Socie G, Devergie A, Girinsky T, Reiffers J, Vernant JP, Le Bourgeois JP, Herve P, Guyotat D, Maraninchi D, Rio B, Michallet M, Jouet JP, Milpied N, Leblond V, Pico J, Attal M, Belanger C, Gluckman E, Cosset JM. Influence of the fractionation of total body irradiation on complications and relapse rate for chronic myelogenous leukemia. Int J Radiation Oncol*Biol*Phys 1991;20(3):397-404.

[40] Devergie A, Blaise D, Attal M, Tigaud JD, Jouet JP, Vernant JP, et al. Allogeneic bone marrow transplantation for chronic myeloid leukemia in first chronic phase: a randomized trial of busulfan-cytoxan versus cytoxan-total body irradiation as preparative regimen: a report from the French Society of Bone Marrow Graft (SFGM). Blood. 1995;85:2263-8.

[41] Magome T, Froelich J, Takahashi Y, Arentsen L, Holtan S, Verneris MR, Brown K, Haga A, Nakagawa K, Holter Chakrabarty JL, Giebel S, Wong J, Dusenbery K, Storme G, Hui SK. Evaluation of Functional Marrow Irradiation Based on Skeletal Marrow Composition Obtained Using Dual-Energy Computed Tomography. Int J Radiation Oncol*Biol*Phys 2016;96(3):679-87.

[42] Magome T, Froelich J, Holtan SG, Takahashi Y, Verneris MR, Brown K, Dusenbery K, Wong J, Hui SK. Whole-Body Distribution of Leukemia and Functional Total Marrow Irradiation Based on FLT-PET and Dual-Energy CT. $153601211773220 \mathrm{Mol}$ Imaging 2017;16. https://doi.org/10.1177/1536012117732203.

[43] Haas B, Coradi T, Scholz M, Kunz P, Huber M, Oppitz U, André L, Lengkeek V, Huyskens D, van Esch A, Reddick R. Automatic segmentation of thoracic and pelvic CT images for radiotherapy planning using implicit anatomic knowledge and organspecific segmentation strategies. Phys Med Biol 2008;53(6):1751-71.

[44] Sharp G, Fritscher KD, Pekar V, Peroni M, Shusharina N, Veeraraghavan H, Yang J. Vision 20/20: Perspectives on automated image segmentation for radiotherapy: Perspectives on automated image segmentation for radiotherapy. Med Phys 2014;41(5):050902. https://doi.org/10.1118/1.4871620.

[45] Liang Y, Kim G-Y, Pawlicki T, Mundt AJ, Mell LK. Feasibility study on dosimetry verification of volumetric-modulated arc therapy-based total marrow irradiation. J Appl Clinical Med Phys 2013;14(2):15-23.

[46] Mancosu P, Navarria P, Castagna L, Reggiori G, Stravato A, Gaudino A, Sarina B, Tomatis S, Scorsetti M. Plan robustness in field junction region from arcs with different patient orientation in total marrow irradiation with VMAT. Phys Med 2015;31(7):677-82. 